\title{
GENERACIÓN, LEGISLACIÓN Y VALORIZACIÓN DE RESIDUOS PLÁSTICOS EN IBEROAMÉRICA
}

\author{
Alethia VÁZQUEZ MORILLAS ${ }^{1,2 *}$, Maribel VELASCO PÉREZ ${ }^{1}$, Rosa Ma. ESPINOSA VALDEMAR ${ }^{1}$, \\ Marcos MORALES CONTRERAS ${ }^{2}$, Saúl HERNÁNDEZ ISLAS ${ }^{2}$, María Yolanda Leonor ORDAZ GUILLÉN ${ }^{3}$ y \\ Hamilcar José ALMEIDA FILGUEIRA ${ }^{4}$
}

${ }^{1}$ Universidad Autónoma Metropolitana, Unidad Azcapotzalco, México Unidad Azcapotzalco Av. San Pablo Núm. 180, Col. Reynosa Tamaulipas, C.P. 02200, México, D.F. Tel. (55) 53189062

${ }^{2}$ Unidad Profesional Interdisciplinaria de Biotecnología, Instituto Politécnico Nacional, México

${ }^{3}$ Centro Interdisciplinario de Investigaciones y Estudios sobre Medio Ambiente y Desarrollo, Instituto Politécnico Nacional, México

${ }^{4}$ Universidad Federal de Paraiba, Brasil

*Autor para correspondencia: alethia@correo.azc.uam.mx

(Recibido diciembre 2014; aceptado julio 2015)

Palabras clave: reciclaje, degradación, bolsas

\section{RESUMEN}

Los plásticos son una de las corrientes de residuos que ha despertado mayor interés en los últimos años, debido a su continuo crecimiento y a sus efectos ambientales, que incluyen la saturación de sitios de disposición final y afectaciones en los ecosistemas, a los que llegan debido a un manejo inadecuado. En esta investigación se analizan y comparan la generación de residuos plásticos no industriales, las medidas legislativas abocadas a la minimización de sus impactos y las tendencias de valorización. La generación de residuos plásticos oscila entre el 9 y el $14 \%$ de la masa de residuos sólidos urbanos, con una tendencia a aumentar en forma directa con el producto interno bruto de los países y en las grandes zonas metropolitanas. Con el fin de dar un manejo integral a estos residuos los países han adoptado medidas que incluyen legislaciones específicas, normas, sistemas de acopio y de información. Un caso relevante, por sus connotaciones ambientales, es el de las bolsas de plástico; las medidas adoptadas a este respecto incluyen su prohibición o la sustitución por bolsas biodegradables. En cuanto a la valorización, se detecta un incremento en el reciclaje, a través del acopio formal e informal. Las medidas establecidas, especialmente en América Latina, han tenido un impacto aún limitado, debido principalmente a la falta de articulación entre las mismas, la producción de plásticos y el sistema de gestión de residuos.

Key words: recycling, degradation, bags

\begin{abstract}
Plastics are one of the waste streams which have attracted increased interest in recent years, due to their continued growth and its environmental effects. Those include saturation of landfills and effects on ecosystems due to littering. This research analyzes and compares the non-industrial plastic waste generation, the environmental policies issued to minimize their environmental impact and the trends in valorization in twelve Ibero-American countries. Generation of plastic waste varies between 9 and $14 \%$. It is directly related to the gross
\end{abstract}


income of the countries and the urbanization in the metropolitan areas. The countries have adopted laws, norms, collecting and information systems in order to manage plastic waste in a sound way. A relevant example, due to its environmental effects, is the case of plastic bags. Strategies such as the restriction of their use or their production from biodegradable materials have been followed in the studied area. Concerning valorization, an increase in recycling can be observed, linked to formal and informal collection systems. These policies, especially in Latin America, have had little impact, due to its lack of integration to the production of plastics and the waste management system.

\section{INTRODUCCIÓN}

La sociedad actual basa su funcionamiento en la utilización de diferentes materiales, entre los cuales destacan los plásticos. Estos comprenden un conjunto amplio de sustancias formadas por polímeros de alto peso molecular, que se producen total o parcialmente en forma sintética, presentan una estructura básica orgánica y a través de manipulaciones químicas o físicas son llevados a un estado sólido final (Schwarz 2002). Sus propiedades pueden variar en función de su composición, pero en general son ligeros, inertes, económicos y presentan una alta relación resistencia - masa. Esto ha llevado a un incremento constante en su producción, que pasó de aproximadamente 99 millones de toneladas en 1989 a 288 millones de toneladas en 2012, con una participación del $4.9 \%$ para Centro y Sudamérica, y de $19.9 \%$ para los países de América del Norte (Plastics Europe 2013).

El aumento en su utilización, su aplicación en productos de vida útil corta y su estabilidad ha llevado a los plásticos a convertirse en una de las corrientes de residuos de mayor crecimiento, lo que ha generado una gran preocupación por parte de la sociedad. En países desarrollados la mayoría de los residuos plásticos son reciclados o entran en procesos de valorización energética (Plastics Europe 2013). Sin embargo, aunque la mayoría de los plásticos que se utilizan en envases y embalajes son reciclables (Hopewell et al. 2009) y existe la tecnología para desarrollar el proceso, en América Latina éste enfrenta obstáculos relacionados con la percepción del material como algo no valioso, la dificultad en su acopio, las fluctuaciones en los precios de los materiales y la carencia de estímulos legales o fiscales (Karak et al. 2012). La valorización energética, por su parte, se ve limitada por los altos costos de inversión y operación, la falta de información y la preocupación por los impactos ambientales que podría generar (Themelis et al. 2013). Por lo anterior, el destino más común para los residuos plásticos en Latinoamérica es su depósito en rellenos sanitarios o tiraderos, en donde se acumulan contribuyendo a la saturación de estos sitios y a los problemas ambientales derivados de los mismos. Cuando no son captados por los sistemas de gestión de residuos, los plásticos se liberan al ambiente, donde afectan a numerosas especies (de Stephanis et al. 2013, Lazar y Gracan 2011, Williams et al. 2011), y contribuyen al transporte y acumulación de contaminantes que se adhieren a su superficie (Browne et al. 2011, Rios et al. 2007).

Las bolsas de un solo uso son los residuos plásticos que han recibido mayor atención por parte de las autoridades y la sociedad en su conjunto. Aunque están presentes en el mercado desde los 40 (Vaughn 2008), fue hasta las últimas décadas que se convirtieron en el medio de transporte de mercancía más empleado en los establecimientos comerciales, debido a su ligereza, resistencia y bajo costo. Se ha estimado que anualmente se consumen en el mundo 6900 millones de las mismas, de los cuales $30 \mathrm{mi}-$ llones llegan al ambiente debido a la disposición no controlada (Equinox Center 2013). Una vez que han sido empleadas para transportar mercancía, es común que las bolsas se utilicen para almacenar artículos o contener los residuos que se desecharán (Ayalon et al. 2009 y Chung 2008). Cuando entran a los sistemas de gestión de residuos su destino varía dependiendo de las prácticas y tecnología disponible en el país; mientras en algunos países se valorizan energéticamente, en otros que carecen de esta opción terminan en rellenos sanitarios, contribuyendo al agotamiento de los mismos.

Cuando las bolsas no se disponen adecuadamente llegan al ambiente, constituyendo entre el 5 y el $20 \%$ de los objetos que son desechados de forma incorrecta (Ayalon et al. 2009). Debido a su bajo peso, son transportadas por el viento, y llegan a los ecosistemas en los que ocasionan daños en diferentes especies (de Stephanis et al. 2013, Müller et al. 2012), además de bloquear drenajes y generar afectaciones estéticas. Aunque diversos análisis de ciclo de vida de bolsas de plástico han planteado que su mayor impacto 
ambiental se da durante su producción y distribución (European Comission-DG Environment 2011, Khoo y Tan 2010), la atención pública se ha centrado en los efectos que generan cuando se convierten en residuos, especialmente en las afectaciones a la vida salvaje. Cuando los plásticos llegan a los ecosistemas, especialmente en sistemas acuáticos, además de adsorber contaminantes hidrofóbicos, pueden liberar algunos de sus aditivos, como ftalatos, bisfenol A y retardantes de flama (Driedger et al. 2015); estos compuestos pueden ingresar a las cadenas tróficas mediante la ingestión por animales marinos y aves (Derraik 2002, Driedger et al. 2015, Van Franeker y Law 2015, Williams et al. 2011).

Lo anterior ha llevado a distintos países como Irlanda, China, Italia, India y Australia a emitir regulaciones que buscan mitigar el impacto de los residuos plásticos en el ambiente (Tolinski 2011), en muchos casos referidos específicamente a las bolsas de plástico de un solo uso. En este artículo se compara la producción de residuos plásticos en 12 países Iberoamericanos, y se revisa la legislación relacionada con estos materiales, con el fin de comparar sus alcances y logros, establecer similitudes y analizar casos de aplicación.
Esta investigación se desarrolló a partir de la revisión de información impresa y electrónica, proveniente de instancias públicas, informes de gobierno, notas de prensa, reportes técnicos, artículos de investigación y documentos generados por asociaciones civiles. Los temas abordados fueron: a) información general sobre el país; b) generación de residuos urbanos; c) generación de residuos plásticos; d) marco legal relacionado con los residuos plásticos; e) medidas de control del uso de bolsas de plástico; f) tendencias en la valorización de residuos plásticos. La información fue recopilada y, siempre que fue posible, se accedió a la fuente original de la misma. Los datos se sistematizaron, ordenaron y analizaron buscando identificar tendencias y ejemplos relevantes.

\section{GENERACIÓN DE RESIDUOS PLÁSTICOS}

En los países estudiados la proporción de plásticos en los residuos sólidos urbanos oscila entre el 9 y el $14 \%$ (Cuadro I), con la mayor generación reportada para Argentina y Panamá. Las diferencias observadas dependen, en gran medida, de los patrones de consumo locales, pero se ven influidas

CUADRO I. GENERACIÓN DE RESIDUOS PLÁSTICOS EN PAÍSES IBEROAMERICANOS

\begin{tabular}{|c|c|c|c|}
\hline País & $\begin{array}{l}\text { Generación per } \\
\text { cápita }(\mathrm{kg} / \mathrm{hab} / \mathrm{año})\end{array}$ & $\begin{array}{l}\% \text { de residuos } \\
\text { plásticos (masa) }\end{array}$ & Referencias \\
\hline Argentina & 0.85 & 14 & $\begin{array}{l}\text { Instituto de Ingeniería Sanitaria (2011), Secretaria de Desarrollo Sustenta- } \\
\text { ble de la Nación (2012) }\end{array}$ \\
\hline Bolivia & 0.43 & 10.0 & Gutiérrez (2009) \\
\hline Brasil & 1.04 & 13.5 & University of Leeds et al. (2014), IPEA (2012) \\
\hline Chile & 1.05 & $9.4-11$ & $\begin{array}{l}\text { Comisión Nacional del Medio Ambiente (2011), Ministerio del Medio } \\
\text { Ambiente (2011) }\end{array}$ \\
\hline Costa Rica & 0.88 & 9.0 & $\begin{array}{l}\text { Instituto Costarricense de Turismo (2013), University of Leeds et al. } \\
\text { (2014) }\end{array}$ \\
\hline España & 0.535 & $10.6^{*}$ & $\begin{array}{l}\text { Asociación española de industriales de plástico (2014), Ministerio de agri- } \\
\text { cultura, alimentación y medio ambiente (2012) }\end{array}$ \\
\hline México & 0.85 & 12.88 & SEMARNAT (2013) \\
\hline Panamá & 0.81 & 14 & Vallester (2010), Autoridad Nacional del Ambiente (2007) \\
\hline Paraguay & 1.04 & $7-10.2$ & OPS y OMS (2011) \\
\hline Perú & 0.61 & $9.48 * *$ & Viceministerio de gestión ambiental (2012) \\
\hline Uruguay & 1.03 & $12-6-13.9$ & $\begin{array}{l}\text { Themelis y Diaz Barriga (2012), Acurio et al. (1998), University of Leeds } \\
\text { et al. (2014) }\end{array}$ \\
\hline Venezuela & 0.85 & $7.6-20 * * *$ & Acuña y Valera (2008), University of Leeds et al. (2014) \\
\hline
\end{tabular}

*Agrupados en la categoría de envases ligeros

** Incluye, además de plásticos, plumas, cenizas y textiles

***Límites mínimo y máximo en ocho de los estados que conforman el país 


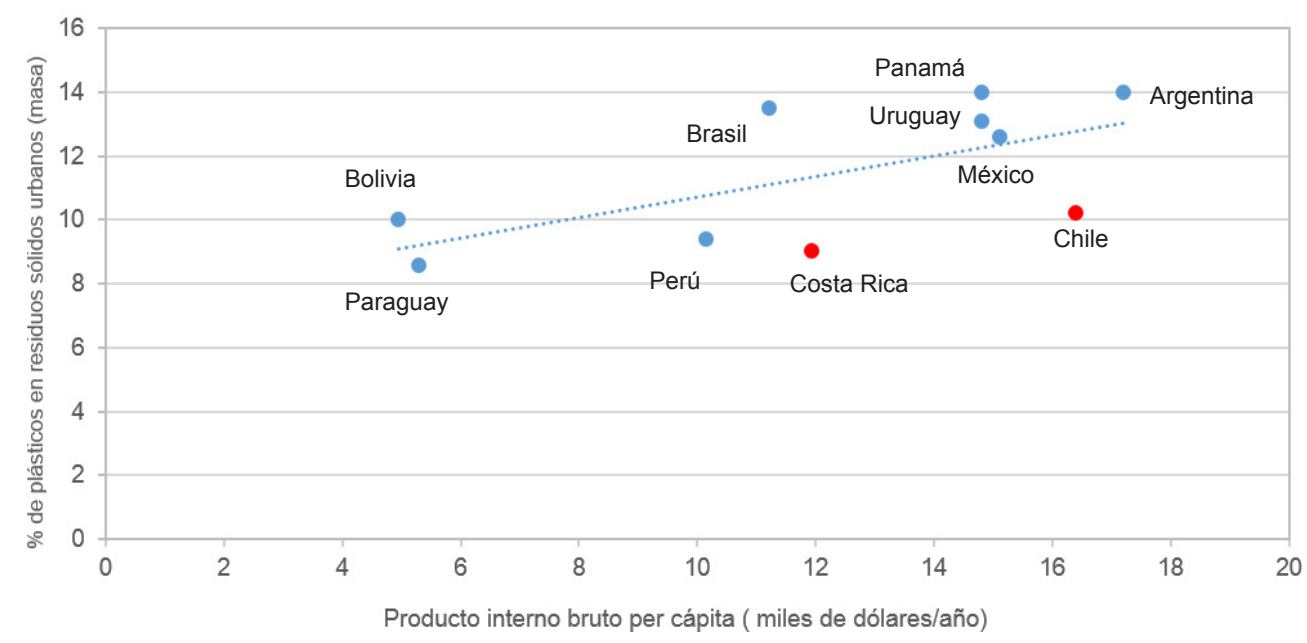

Fig. 1. Relación entre producto interno bruto y proporción de plásticos en los residuos

también por la fecha de realización de los estudios, variaciones en las metodologías de medición, por la inclusión o exclusión de residuos comerciales dentro de los residuos urbanos, por los tipos de materiales considerados en cada caso en la categoría de residuos plásticos y por la existencia de actividades formarles e informales de recolección. Generalmente ésta agrupa a las bolsas, películas, artículos desechables y envases rígidos; los hules, por su parte, no siempre son incluidos, y lo mismo ocurre con residuos que parcialmente están compuestos de plástico, como los pañales desechables, juguetes, artículos electrónicos y para el hogar. Por lo anterior puede inferirse que la presencia de plásticos en los residuos puede ser mayor a los valores reportados.

Con frecuencia se considera que un mayor desarrollo económico conduce a un incremento en la generación de residuos (Bandara et al. 2007, Osinga et al. 2011) y que, al mismo tiempo, el aumento en el poder adquisitivo incrementa modifica los patrones de consumo (Troschinetz y Mihelcic 2009), lo que podría provocar un aumento en la proporción de materiales plásticos. Sin embargo, en este caso no se observa correspondencia entre la generación per cápita y la proporción de residuos plásticos. Por otro lado, se puede inferir cierta relación entre el desarrollo económico y la proporción de residuos plásticos (Figura 1). Sin embargo, se observan excepciones, como los casos de Costa Rica y Chile, que presentan alto PIB y baja proporción de residuos plásticos. Este desacoplamiento puede deberse a los diferentes niveles de industrialización y desarrollo de los países (Troschinetz y Mihelcic 2009), así como a factores culturales. El análisis de los datos permite corroborar la relación entre la producción de los residuos plásticos y el nivel de urbanización de las ciudades. En el cuadro II se compara la generación nacional con la de algunas de las megaciudades latinoamericanas; pueden observarse incrementos de hasta tres puntos porcentuales con relación a los promedios de los respectivos países.

La carga que los residuos plásticos representan al ambiente es función tanto de su proporción en los residuos sólidos urbanos como de la generación total. A este respecto, se observa una mayor generación per cápita en los países de mayor desarrollo, con diferencias de hasta $300 \%$ entre Brasil y Bolivia (figura 2). Además de los impactos ambientales debidos a la disposición no controlada de estos residuos, debe considerarse su contribución al agotamiento de los rellenos y vertederos; dado que los plásticos son materiales de muy baja densidad, su proporción volumétrica en los sistemas de manejo de residuos es hasta 2.5 veces mayor que la proporción en masa, es decir, entre $25 \mathrm{y}$ 35 \% (Barlow y Morgan 2013).

\section{LEGISLACIÓN SOBRE RESIDUOS PLÁSTICOS}

El marco regulatorio sobre residuos plásticos varía en amplitud y estructura en los diferentes países. En general se les identifica como uno de los componentes de los residuos sólidos urbanos, por lo que están sujetos a las disposiciones que aplican a los mismos. Argentina (CEAMSE 2014), Brasil (Presidencia de la República 2010), México (SEMARNAT 2003), España (Consejo de Ministros 2009), Paraguay 
CUADRO II. PROPORCIÓN DE RESIDUOS PLÁSTICOS EN MEGACIUDADES LATINOAMERICANAs

\begin{tabular}{|c|c|c|c|c|}
\hline Ciudad & $\begin{array}{l}\text { Generación per } \\
\text { cápita }(\mathrm{kg} / \mathrm{hab}- \\
\text { día) }\end{array}$ & $\begin{array}{l}\text { \% local de residuos } \\
\text { plásticos (masa) }\end{array}$ & $\begin{array}{l}\% \text { nacional de } \\
\text { residuos plásticos } \\
\text { (masa) }\end{array}$ & Referencias \\
\hline $\begin{array}{l}\text { Buenos Aires, } \\
\text { Argentina }\end{array}$ & 1.52 & 18.54 & 14 & $\begin{array}{l}\text { Instituto de Ingeniería Sanitaria (2011), } \\
\text { Observatorio Nacional para la Gestión de } \\
\text { Residuos Sólidos Urbanos (2009) }\end{array}$ \\
\hline $\begin{array}{l}\text { Río de Janeiro, } \\
\text { Brasil }\end{array}$ & 1.6 & $16.5-23.7$ & 13.5 & $\begin{array}{l}\text { Münnich et al. (2006), United Nations } \\
\text { Environment Programme (n.d.) }\end{array}$ \\
\hline $\begin{array}{l}\text { Zona metropolitana } \\
\text { de Santiago, Chile }\end{array}$ & 1.05 & 10.07 & $9.4-11$ & $\begin{array}{l}\text { Grupo de Residuos Sólidos (2006), Comi- } \\
\text { sión Nacional del Medio Ambiente (2010). }\end{array}$ \\
\hline $\begin{array}{l}\text { Distrito Federal, } \\
\text { México }\end{array}$ & 1.45 & 13.6 & 12.88 & Durán et al. (2013) \\
\hline Lima, Perú & 0.64 & 11.01 & 9.48 & $\begin{array}{l}\text { Dulante (2013) Vicemministerio de Gestión } \\
\text { Ambiental (2012). }\end{array}$ \\
\hline
\end{tabular}

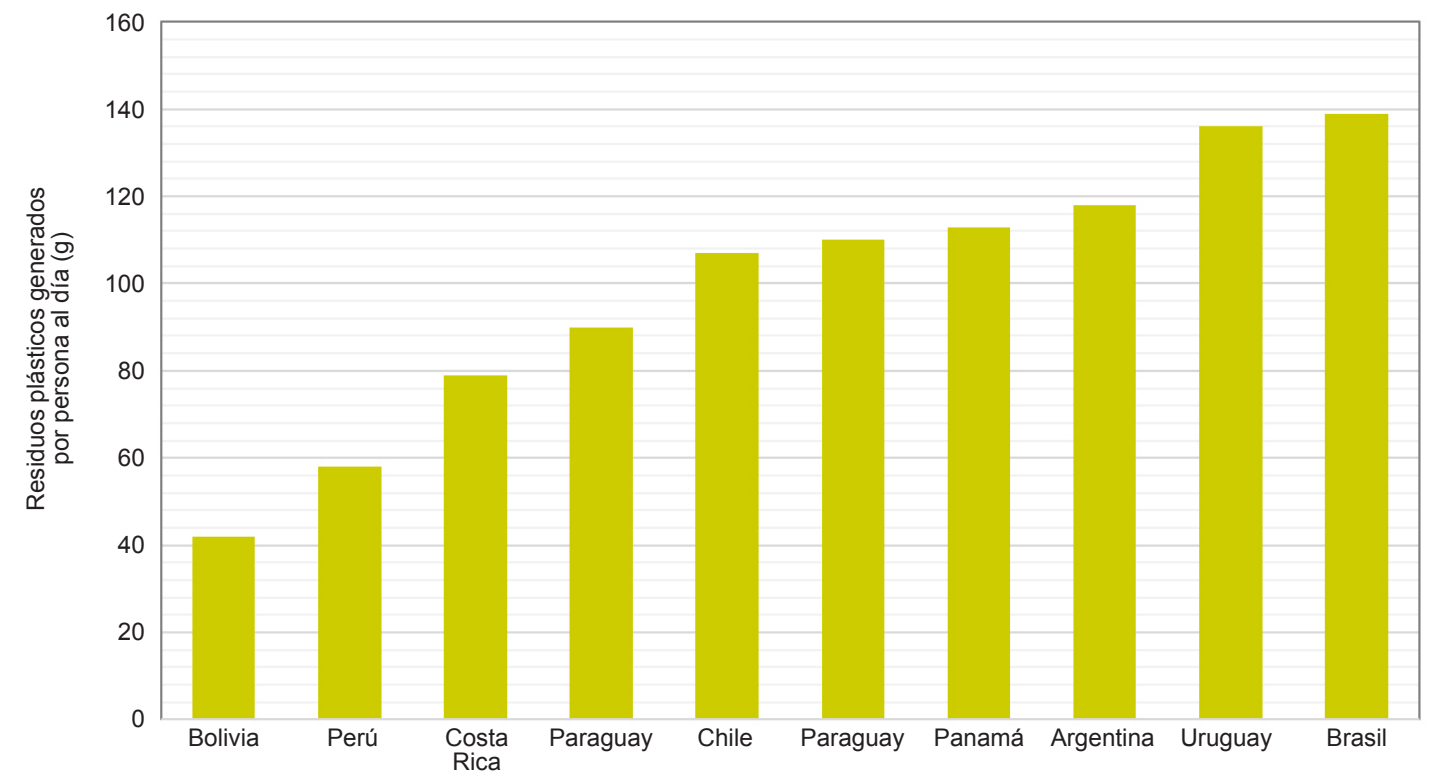

Fig. 2. Generación de residuos sólidos per cápita en países latinoamericanos

(Congreso de la Nación Paraguaya 2011) y Venezuela (Asamblea Nacional de la República Bolivariana de Venezuela 2010) cuentan con leyes o políticas nacionales de residuos recientes, por lo que en general sus principios coinciden con las prácticas actuales de gestión integral de residuos. Así, en ellas se promueve la articulación de funciones entre los distintos órdenes de gobierno, la jerarquía de las opciones de manejo de residuos (3 R's) y la valorización de los mismos. Destaca, en este contexto, el caso de España, que debido a su pertenencia a la Unión Europea, debe acatar las disposiciones de la Directiva marco de residuos (UE 2008), que establecen metas concretas de recuperación y reciclaje de residuos, entre los cuáles se encuentran los envases plásticos, así como la Directiva sobre residuos de aparatos eléctricos y electrónicos (que contienen plásticos), que incluye lineamientos sobre acopio, tratamiento y valorización (UE 2003). Panamá y Uruguay carecen de legislaciones integrales sobre residuos, y su normativa está basada en la emisión de decretos.

El manejo directo de los residuos plásticos recae, en todos los casos, en las autoridades de los municipios o ayuntamientos. Esto genera dificultades al momento de aplicar las disposiciones generales establecidas en las leyes nacionales, lo que se ve reflejado en los niveles bajos de recuperación que se tiene en comparación con países de otras latitudes. 
La legislación dirigida específicamente a los residuos plásticos difiere de un país a otro, en los casos en que existe. A continuación se describen y ejemplifican los diferentes elementos normativos que se han generado en la región con el fin de promover la valorización de los residuos plásticos.

\section{Leyes especificas sobre residuos plásticos}

Uruguay cuenta con una Ley de uso de envases no retornables, la cual regula su identificación y uso, a la vez que obliga al registro de productores y al desarrollo de planes de manejo por parte de los mismos (Senado y Cámara de Representantes de la República Oriental de Uruguay 2004). Como medida complementaria, se emitió un decreto que obliga a las instancias públicas a realizar sus compras en empresas registradas en dicho padrón (Presidencia de la república de Uruguay 2010). En Argentina se presentó en 2009 un proyecto de ley sobre un Sistema de Gestión de Envases y Residuos de Envases (Romero 2014), que tiene como objetivo prevenir y reducir el impacto sobre el medio ambiente de los envases y la gestión de los residuos de envases, a través de una gestión integral de los mismos considerando su ciclo de vida. Esta medida incluye aspectos de producción sustentable, valorización, eliminación de materiales nocivos, responsabilidad extendida del productor y aranceles favorables para la reutilización; establece metas de valorización, reducción y reciclaje, así como la necesidad de crear un sistema integrado de gestión de residuos.

\section{Acopio de residuos plásticos para su valorización}

Además de lo establecido en las Leyes mencionadas previamente, países como Brasil (Presidencia de la República 2006) y Venezuela (Presidencia de la República 1992) han emitido decretos sobre la separación en la fuente de residuos reciclables, específicos para los órganos de la administración pública, en el primer caso, y generales en el segundo.

Una segunda aproximación es el reconocimiento de la aportación de los recicladores informales a la valorización de los residuos plásticos. Al dotar de un marco jurídico se facilita la incorporación de estas agrupaciones o individuos a los sistemas formales de gestión de residuos, lo que permite, además de mejorar sus condiciones de vida, hacer más eficiente y regular el acopio de residuos valorizables. Este enfoque se ha probado con éxito en Chile, Perú y Brasil, a través de sus políticas de inclusión de recicladores de base (OIY y AVINA 2013, Presidencia de la República de Perú 2010a, Terraza y Sturzenegger 2010).

\section{Normas técnicas y ambientales}

Los organismos autónomos de normalización de Argentina y México han emitido normas relacionadas con la terminología de bioplásticos, plásticos degradables y compostables, cuyo fin último es la certificación de materiales que ostentan esas denominaciones. En el caso de Argentina, se cuenta también con una norma que establece requisitos para plásticos biodegradables o compostables (IRAM 2009, IRAM 2011) y se tiene el proyecto de emitir normas que describan los métodos de prueba que se derivan de dichos requisitos. En México existe una norma sobre terminología de Bioplásticos (SEGOB 2014). Para ambos países estas normas surgieron de asociaciones civiles que generan normas a partir de las necesidades detectadas en las industrias y mercados locales, buscando al mismo tiempo la similitud con las normas ISO equivalentes.

En México el gobierno del Distrito Federal emitió un conjunto de criterios y normas de producción y consumo sustentable de los productos plásticos, en los cuales se orienta sobre la forma de reducir impactos ambientales durante la fabricación, y se plantean lineamientos sobre las características deseables en las bolsas de plástico (SEDEMA 2011). A nivel federal se cuenta con una norma para el manejo de residuos considerados "de manejo especial", entre los cuales se incluyen envases, películas y otros residuos que están compuestos total o parcialmente de plástico, como los electrónicos. En esta norma se establece la necesidad de generar planes de manejo que permitan valorizar estos residuos, así como los mecanismos para su puesta en marcha y seguimiento (SEMARNAT 2011).

En el caso de España se cuenta con normas técnicas sobre vocabulario, clasificación, identificación y reciclaje para diferentes plásticos y envases. Estas son equivalentes a las normas europeas "EN" sobre cada uno de los temas (Ministerio de Fomento et al. 2013).

\section{Sistemas de información}

Chile ha desarrollado el Sistema Nacional de Declaración de Residuos Sólidos (SINADER), a través del cual generadores y destinatarios de residuos (industrias y municipios) deben reportar la generación y caracterización de los desechos. Entre los productos que debe identificarse se encuentran los residuos plásticos, con lo que se establecen las bases para la generación de inventarios nacionales que permitan una mejor gestión y aprovechamiento (Ministerio del Medio Ambiente 2014). En México se ha avanzado en el registro del manejo integral de algunas corrientes 
de residuos plásticos, mediante la obligatoriedad del registro de los planes de manejo para los residuos de manejo especial que establece la norma mencionada previamente (SEMARNAT 2011).

\section{EL CASO DE LAS BOLSAS DE PLÁSTICO}

En el ámbito internacional las medidas aplicadas para regular la utilización de bolsas de plástico implican generalmente la aplicación de impuestos o la prohibición o limitación del uso de bolsas, que con frecuencia va aparejada a la obligatoriedad del uso de bolsas con características específicas.

La aplicación de impuestos, mediante la cual el consumidor tiene que pagar una tasa por la adquisición de las bolsas de plástico de un solo uso, se ha aplicado en países como Irlanda, Taiwan, Bélgica y Dinamarca (Freinkel y Frainkel 2011). En Iberoamérica se ha optado en general por las medidas que buscan la disminución en el uso de bolsas de plástico, que muestran dos distintas vertientes: la disminución progresiva en el uso de bolsas de plástico, hasta su eliminación, y la búsqueda de su sustitución por bolsas hechas de plásticos biodegradables o material reciclado (por ejemplo en México, las normas de producción y consumo sustentable de los productos plásticos a nivel estatal, en el Distrito Federal). Aunque se han promovido leyes a nivel nacional, en la mayoría de los casos las propuestas han sido locales, generando una gran atención mediática y por parte de los ciudadanos.

\section{Eliminación de bolsas}

En lo referente a la eliminación de las bolsas plásticas, en Perú se presentó el proyecto de Ley para la prevención y control de los riesgos del uso de las bolsas plásticas en mercados y supermercados, que tiene como objeto proteger a la persona, la comunidad y el medio ambiente en el que se desarrollan de las consecuencias sanitarias del uso descontrolado de las bolsas plásticas, y que el uso de las mismas sea responsable, sólo en las ocasiones que lo ameriten. La ley prohíbe su uso, y en caso indispensable, obliga a contener mensajes que mencionen sus consecuencias ambientales (Congreso de la República de Perú 2013). En Chile se encuentra a discusión el proyecto de Ley mediante el cual se prohíbe, dentro del territorio de la Patagonia Chilena, la entrega, a cualquier título, de bolsas plásticas no biodegradables y biodegradables que utilizan los clientes para la contención y transporte de mercaderías en supermercados, almacenes, tiendas, kioscos y demás establecimientos de comercio (Comisión de zonas extremas y Antártica Chilena 2014).

En Venezuela, aunque no se han prohibido las bolsas, se han generado ordenanzas municipales tendientes a restringir el uso de bolsas plásticas con capacidad menor a $30 \mathrm{~kg}$ (Masverde 2011, Scott 2011).

\section{Sustitución por bolsas biodegradables}

La sustitución de las bolsas convencionales, generalmente fabricadas a partir de polietileno, por bolsas biodegradables, es una medida controversial que se ha implementado en diferentes ciudades Iberoamericanas, generalmente con efectos limitados en términos de beneficio ambiental. La mayor complicación consiste en la necesidad de generar todo un marco regulatorio para sustentar esta decisión, de forma que los posibles beneficios ambientales de la tecnología puedan aprovecharse. Lo anterior se puede ilustrar al comparar los casos de Buenos Aires, en Argentina, y del Distrito Federal, en México.

En Buenos Aires se aprobó en 2007, la Ley $\mathrm{N}^{\mathrm{o}}$ 1.854, que establece como obligatorio el uso de bolsas biodegradables para la recolección de los residuos sólidos urbanos húmedos, así como el uso de materiales biodegradables (Legislatura de la Ciudad Autónoma de Buenos Aires 2005).

Esta medida fue seguida en los años posteriores por decretos, resoluciones y leyes que definen las competencias de las distintas instancias involucradas en su aplicación, la forma en que se evaluará la biodegradabilidad de los materiales (a partir de normas técnicas generadas para tal fin) y se establece un Plan para dar cumplimiento a la Ley, tal como se muestra en el cuadro III. En el Distrito Federal, por otro lado, en 2009 la Asamblea Legislativa del Distrito Federal modificó la Ley de Residuos (GDF 2009), estableciendo la prohibición de que los establecimientos comerciales proporcionasen a sus clientes bolsas de plástico, a menos que éstas fueran biodegradables. Durante el año previo a su aplicación esta reforma fue ampliamente discutida, tanto en lo referente a su conveniencia como en lo que se consideraría como plástico biodegradable. Se instaló un grupo de trabajo para generar una norma que permitiese evaluar la biodegradabilidad de las bolsas, pero la discusión no logró llevar a un acuerdo inicial sobre los tipos de plástico que deberían entrar en la categoría de biodegradable. Esto, aunado a la presión ejercida por parte de la industria del plástico, provocó que en 2010 (GDF 2010) la Ley se modificara nuevamente, esta vez para eliminar la prohibición original y recomendar el uso de bolsas biodegradables o de aquellas que contienen aditivos que provoquen su desintegración 
CUADRO III. INSTRUMENTOS PARA LA REGULACIÓN DE LAS BOLSAS DE PLÁSTICO EN BUENOS AIRES, ARGENTINA

\begin{tabular}{|c|c|}
\hline Año & Instrumento regulatorio \\
\hline 2007 & $\begin{array}{l}\text { Ley } \mathrm{N}^{\mathrm{o}} 1.854 \text { establece como obligatorio el uso de bolsas biodegradables para la recolección de los residuos sólidos } \\
\text { urbanos húmedos, así como el uso de materiales biodegradables }\end{array}$ \\
\hline 2007 & $\begin{array}{l}\text { Resolución } N^{\circ} \text { 857-MMAGC/07, que define como bolsas biodegradables a aquellas que son compostables de acuer- } \\
\text { do al término definido en la norma IRAM-ISO 14.021:2000 o la norma que la reemplace }\end{array}$ \\
\hline 2009 & $\begin{array}{l}\text { Ley } \mathrm{N}^{\circ} 3.147 \text {, que tiene como objeto fomentar el desarrollo de la producción de bolsas biodegradables; la reducción } \\
\text { progresiva y posterior prohibición en la entrega de bolsas no biodegradables por parte de los comercios; y la sustitu- } \\
\text { ción de sobres y bolsas no biodegradables por aquellos que sí lo son (Aires 2009). }\end{array}$ \\
\hline 2012 & $\begin{array}{l}\text { Decreto 180/2012, en el que se designa a la Agencia de Protección Ambiental como autoridad para la aplicación de } \\
\text { la Ley } N^{\circ} 3.147 \text {, y la instruye para elaborar y difundir un "Plan de Reducción de Bolsas y de Sustitución de Sobres } \\
\text { no biodegradables (Poder Ejecutivo 2012). }\end{array}$ \\
\hline 2012 & $\begin{array}{l}\text { Resolución N } 155 / \text { APRA/2012por la cual se aprueba el Plan de Reducción de Bolsas y Sustitución de Sobres No } \\
\text { Biodegradables }\end{array}$ \\
\hline 2012 & $\begin{array}{l}\text { Resolución 193/2012 que establece modificaciones a la resolución anterior relacionadas con el tipo de verificación y } \\
\text { certificación de las normas, utilización de colores en las bolsas y mensajes }\end{array}$ \\
\hline
\end{tabular}

en un periodo menor a los 5 años, haciendo una clara alusión a los plásticos oxodegradables. Lo último provocó un aumento de la presencia de estos últimos en el mercado, aunque no existe en la actualidad un mecanismo para evaluar que las afirmaciones sobre el uso de cierta tecnología en la fabricación de bolsas tengan sustento.

Entre los países que han propuesto regulaciones nacionales que promueven el uso de bolsas biodegradables está Perú, en el que la Comisión de Pueblos Andinos, Amazónicos, Afroperuanos Ambiente y Ecología del Congreso presentó y discutió el Predictamen del proyecto de Ley de Promoción del Uso Responsable de Bolsas de Plástico. El objetivo del proyecto es impulsar el uso gradual de bolsas biodegradables, buscando reducir el impacto ambiental que generan las bolsas de plástico convencionales que son ofrecidas a los consumidores en miles de establecimientos comerciales en todo el país. Para ello, se generaría primero una norma técnica que defina qué son bolsas biodegradables, oxobiodegradables o compostables, por el vacío normativo y conceptual que hay al respecto (Niezen 2014). Por su parte, en España, la Ley de residuos y suelos contaminados indica que al 2015 se debe alcanzar la sustitución del $70 \%$ de las bolsas comerciales de un solo uso de plástico no biodegradable, que a partir de entonces deberán llevar un mensaje con sus efectos ambientales. El objetivo es eliminar su utilización antes del 2018 (Jefatura del Estado 2011), con el fin de ajustarse a los lineamientos de la Comunidad Europea. Para hacerlo, en el país es vigente la norma europea EN 13432:2000 (CEN 2000), que permite evaluar la biodegradabilidad de los plásticos en condiciones de composteo. En Paraguay se presentó en la cámara de senadores el proyecto de ley "De promoción a la conveniencia saludable y ambiental en Paraguay a través de la disminución del uso de plástico polietileno", que busca su reemplazo progresivo por materiales degradables y/o biodegradables. Además de estas medidas a nivel nacional se han promovido regulaciones locales en numerosas ciudades o regiones, que incluyen Pucón, en Chile (Municipalidad de Pucón 2013), Neuquén, Mendoza y Santa Cruz, en Argentina (Consejo Deliberante de Neuquén 2014), Quintana Roo y Boca del Río en México (De la Tijera 2010), sólo por mencionar algunos ejemplos.

\section{Compras verdes}

Las medidas que buscan promover el uso de bolsas biodegradables o fabricadas a partir de materiales reciclados se han reforzado con el establecimiento de políticas de compras verdes. El gobierno de Perú emitió un decreto por medio del cual se obliga al sector público a utilizar plásticos, papeles y cartones con $80 \%$ de material de reciclado, así como a la compra y uso obligatorio de bolsas de plástico biodegradables (Presidencia de la República de Perú 2010b).

\section{VALORIZACIÓN DE RESIDUOS PLÁSTICOS}

Con excepción de lo que ocurre en España, en Latinoamérica la valorización de residuos plásticos ha ocurrido casi exclusivamente a través del reciclaje me- 
cánico de los mismos, que implica transformaciones físicas de los residuos que no alteran su composición química. Las tasas de valorización de residuos plásticos, en los casos en que se reportan, presentan variaciones importantes, que pueden atribuirse en parte a la forma en que se mide el reciclaje; mientras que en algunos casos se reporta la contribución de los plásticos en las corrientes de residuos reciclados, en otros se mide la proporción de residuos urbanos plásticos reciclados. En general, debido a la preponderancia de la recolección informal de estos residuos, su cuantificación resulta compleja.

Las cifras reportadas varían desde el $2 \%$ de residuos plásticos reciclados reportado para Venezuela (Instituto Nacional de Estadística 2006) hasta el $21.7 \%$ que se registra para Brasil (Plastivida 2011). Chile, Argentina y México han reportado cifras de 4, 10 y $11 \%$, respectivamente (INEGI 2012, Ministerio de Medio Ambiente 2011). En Perú, la información oficial indica que al 2013, 205 municipalidades implementaron programas de segregación en la fuente, con lo que se recuperaron para reciclar 10974 ton/mes (Ministerio del Ambiente 2014). En España, por otro lado, los residuos de envases de plástico se reciclaron y valorizaron en un 32 y $56 \%$, respectivamente, en 2011. Con eso se cumplió la Directiva Europea 94/62/ CE que marcaba una meta de $22.5 \%$ de reciclaje en 2008 (Ministerio de Agricultura Alimentación y Medio Ambiente 2014). En la mayoría de los países se reporta un incremento en el número de empresas dedicadas al reciclaje. Otro factor común es el hecho de que el acopio de los residuos plásticos se realiza a través de sistemas de recolección separada y puntos de acopio, pero sobre todo, de la recolección informal (Gutberlet y Baedera 2008, Medina 2000, Medina 2005).

Mientras que en España los materiales más reciclados fueron los polietilenos (PEBD $29 \%$, PEAD, $24 \%$ ) (ECOEMBRES 2012), en Brasil y México el plástico de origen doméstico más valorizado es el PET (Associação Brasileira da Indústria do PET 2012, PETSTAR n.d.). En los casos que se presenta mayor avance se ha tenido una fuerte colaboración con la industria de los envases y asociaciones civiles. Un ejemplo de lo anterior tiene lugar en Santiago, Chile, donde se generó en 2008 la Iniciativa Santiago Recicla, liderada por la Dirección Regional Metropolitana de la CONAMA, la Intendencia Metropolitana y la Fundación Casa de la Paz. A través de ella se estableció una mesa de trabajo constituida por representantes de instituciones públicas, privadas y de la sociedad civil, con el fin de conocer y desarrollar acciones para aumentar la tasa regional de reciclaje. Una de sus metas fue aumentar el reciclaje de 14 a
$25 \%$ para el 2020 , mediante la propuesta de construcción de 10 centros de valorización y 100 puntos de recuperación, que incluyen botellas de plástico. $\mathrm{Su}$ plan de acción menciona la existencia de al menos una empresa recicladora de PET, una de bolsas y una de tapas de botellas en la zona (CONAMA et al. 2009). En Argentina se conformó ECOPLAS, una asociación técnica profesional especializada en plásticos y medio ambiente. Creó y lleva a cabo el programa Polietileno reciclable, para la gestión de los envases plásticos post-consumo, en el cual se identifica con un logo a los envases compatibles con la cadena de la industria del reciclado del polietileno (ECOPLAS 2014). En México el exitoso reciclaje de PET, a través de sistemas botella a botella, tuvo su origen en la conformación en 2002 de una asociación civil, ECOCE, que estableció precios de garantía y dio viabilidad al acopio de este material (PETSTAR n.d.).

\section{CONCLUSIONES}

Los plásticos constituyen una corriente de residuos de atención prioritaria en Iberoamérica, tanto por su generación como por la posibilidad de valorizarlos. Esta relevancia se ha visto reflejada en iniciativas para su acopio y reciclaje, tanto de los gobiernos locales como de la industria y la sociedad civil.

Se puede observar una tendencia a la inclusión de elementos de gestión integral de residuos plásticos en la legislación ambiental de los distintos países. Sin embargo, el análisis de la normatividad aplicada a los mismos pone en evidencia la falta de articulación entre las diferentes medidas, que disminuye la posibilidad de aplicarlas de manera efectiva. Las autoridades que emiten estas normas y leyes generalmente no realizan evaluaciones sobre su aplicación, por lo que no es posible medir de manera objetiva su impacto.

Las estrategias aplicadas en distintos países buscan la promoción del reciclaje o el uso de materias primas con menos impactos ambientales. Aunque se han dado los primeros pasos es necesario afrontar retos significativos, como la dispersión de los mercados, el hecho de que generalmente involucren a muchas pequeñas empresas o el que no se cuente con organismos que avalen la biodegradabilidad o contenido reciclado de estos materiales, con lo que es complejo dar seguimiento a las medidas.

Pueden detectarse, en los casos analizados, elementos que contribuyen al éxito de las iniciativas de valorización. La más relevante es la colaboración de las autoridades con los sectores productivo y 
social, que permite la articulación de las medidas legislativas con los elementos de mercado, dando sentido ambiental y económico al reciclaje. Aunque probablemente esto no sea posible para todos los residuos plásticos, resulta una medida viable en aquellos destinados al envase y embalaje, que constituyen una proporción significativa. Otro elemento a destacar es la generación de marcos normativos completos e integrales, como el planteado para las bolsas en Argentina, que pueden servir como modelo para otras regiones.

Aunque no se aborda en esta investigación, no debe obviarse la relevancia de la participación social y de las medidas de responsabilidad extendida en el manejo de los residuos plásticos. Finalmente, debe tenerse en cuenta que una gestión integral y un marco legislativo consistente deben contemplar no sólo los efectos que generan como residuos, sino el ciclo de vida completo de los plásticos, materiales insustituibles en la vida moderna.

\section{AGRADECIMIENTOS}

Los autores agradecen la posibilidad de realizar este trabajo conjunto entre diferentes países iberoamericanos a CYTED (Proyecto 715RT0494).

\section{REFERENCIAS}

Acuña S., y Valera, V. (2008). Indicadores de residuos y desechos sólidos en Venezuela. En I Simposio Iberoamericano de Ingeniería de Residuos, Castellón, 23-24 de junio de 2008.

Acurio G., Rossin A., Taixeira P. F., y Zepeda, F. (1998). Diagnóstico de la situación del manejo de residuos sólidos municipales en América Latina y el Caribe. Publicación conjunta del Banco Interamericano de Desarrollo y la Organización Panamericana. Washington, D. C. $130 \mathrm{pp}$.

Agencia de protección ambiental (2012). Resolución 155/2012 Aprobación del Plan de reducción de bolsas y sustitución de sobres no biodegradables. Publicado el 9 de mayo de 2012. Buenos Aires, 113 pp.

Aires L. de la C. A. de B. Ley No. 3147/09 (2009).

Asamblea Nacional de la República Bolivariana de Venezuela (2010). Ley de gestión integral de la basura. Publicada en la Gaceta Oficial el 30 de diciembre de 2010. Caracas, $10 \mathrm{pp}$.

Asociación española de industriales de plásticos (2014). Preguntas frecuentes. [en línea]. http://www.anaip.es/ escaparate/verpagina.cgi?idpagina $=203140$ 14/04/ 2014
Associação Brasileira da Indústria do PET (2012). 9o CENSO da Reciclagem de PET - Brasil. O 2012. Reporte. [en línea]. http://www.abipet.org.br/index. $\mathrm{html}$ ? method $=$ mostrarDownloads\&categoria $. i \mathrm{~d}=3$ $3 / 03 / 2014$

Autoridad Nacional del Ambiente (2007). Taller sobre el modelo de emisiones en relleno sanitario para la región Centroamericana. El Salvador, marzo 2007.

Ayalon O., Goldratt T., Rosenthal, G. y Grossman M. (2009). Reduction of plastic carrier bag use: An analysis of alternatives in Israel. Waste Manage. 29 (7), 2025-32. http://dx.doi.org/10.1016/j.wasman.2009.02.016

Bandara N. J., Hettiaratchi J. P. A., Wirasinghe S. C. y Pilapiiya S. (2007). Relation of waste generation and composition to socio-economic factors: a case study. Environ. Monit. Assess. 135 (1-3), 31-39 DOI:10.1007/s10661-007-9705-3

Barlow C. Y. y Morgan D. C. (2013). Polymer film packaging for food: An environmental assessment. Resour. Conserv. Recy. 78, 74-80. http://dx.doi.org/10.1016/j. resconrec.2013.07.003

Browne M. A., Crump P., Niven S. J., Teuten E., Tonkin A., Galloway T. y Thompson R. (2011). Accumulation of Microplastic on Shorelines Worldwide: Sources and Sinks. Environ. Sci. Technol. 45 (21), 9175-9179. DOI: $10.1021 / \mathrm{es} 201811 \mathrm{~s}$

Chung S.S. (2008). Using plastic bag waste to assess the reliability of self-reported waste disposal data. Waste Manage. 28 (12), 2574-84. http://dx.doi.org/10.1016/j. wasman.2008.01.002

Comisión de zonas extremas y Antártica Chilena (2014). Boletín No. 1933-12-2 - Segundo informe de la comisión de zonas extremas y Antártica Chilena, recaído en el proyecto de Ley que establece la prohibición y sustitución progresiva de las bolsas de polietileno, polipropileno y otros polímeros artificiales. [en línea]. http://www.camara.cl/trabajamos/sesion_ordendia. aspx?prmid=3069 10/08/ 2014

Comisión Nacional del Medio Ambiente (2010). Primer reporte sobre manejo de residuos sólidos en Chile. Reporte. Santiago de Chile, 64 pp.

Congreso de la Nación Paraguaya (2011). Ley No 3956 Gestión integral de los residuos sólidos en la República del Paraguay. Asunción, 10 pp.

Congreso de la República de Perú (2013). Proyecto de ley No 3208/2013 - Proyecto de ley para la prevención y control de los riesgos del uso de las bolsas plásticas en mercados y supermercados. Lima, 9 pp.

Consejo de Ministros (2009). Plan Nacional Integrado de Residuos para el periodo 2008-2015. Publicado en el Boletín Oficial Del Estado el 26 de febrero de 2009. Madrid, 124 pp. 
Consejo Deliberante de Neuquén (2014). Ordenanza 12.962. Aprobada el 3 de abril de 2014. Neuquén, Argentina.

CEAMSE (2014). Ley 25.916 de Gestión de residuos domiciliarios. Coordinación Ecológica del área metropolitana Sociedad Argentina. Legislación nacional. Buenos Aires, 6 pp.

CEN (2000). EN 13432 Packaging. Requirements for packaging recoverable through composting and biodegradation. Test scheme and evaluation criteria for the final acceptance of packaging. Comité Europeo de Normalización.

CONAMA, Intendencia Región Metropolitana de Santiago y Fundación Casa de la Paz (2009). Plan de acción de reciclaje, mesa intersectorial "Santiago Recicla", región metropolitana, Versión 6.0. Reporte. Santiago de Chile, 25 pp.

De la Tijera E. (2010). Regulaciones y estándares ambientales sobre bolsas de plástico. Foros Sobre La Sustentabilidad de Los Plásticos, 16-19 de marzo de 2010. México, D. F.

De Stephanis R., Giménez, J., Carpinelli E., GutierrezExposito C., y Cañadas A. (2013). As main meal for sperm whales: Plastics debris. Mar. Pollut. Bull. 69 (1), 206-214. http://dx.doi.org/10.1016/j.marpolbul.2013.01.033

Derraik J. (2002). The pollution of the marine environment by plastic debris: a review. Mar. Pollut. Bull. 44 (9) 847-852. http://dx.doi.org/10.1016/S0025326X(02)00220-5

Driedger A., Dürr H., Mitchell K. y Van Cappellen P. (2015). Plastic debris in the Laurentian Great Lakes: a review. Journal of Great Lakes Research 41 (1), 9-19. http://dx.doi.org/10.1016/j.jglr.2014.12.020

Dulante A. (2013). Asignación de competencias en materia de residuos sólidos de ámbito municipal y sus impactos en el ambiente. Tesis de licenciatura, Universidad Pontificia Católica del Perú. Lima, Perú.

Durán A., Garcés M., Velasco A. R., Marín J. C., Gutiérrez R., Moreno A. y Delgadillo N. A. (2013). Mexico City's municipal solid waste characteristics and composition analysis. Revista Internacional de Contaminación Ambiental, 29 (1), 39-46.

ECOEMBES (2012). El reciclaje de envases domésticos en España. [en línea]. http://www.ecoembes.com/sites/ default/files/resultados_2012.pdf 3/03/2014.

ECOPLAS (2014). Programa Polietileno Reciclable de ECOPLAS. Boletín Técnico Informativo. Salguero, Argentina, $11 \mathrm{pp}$.

Equinox Center (2013). Plastic bag bans: analysis of economic and environmental impacts. Reporte. San Diego, California, 35 pp.

European Commission-DG Environment (2011). Assessment of impacts of options to reduce the use of single-use plastic carrier bags. Reporte. París, 135 pp.
Freinkel, S., y Frainkel, S. (2011). Plastic. A toxic love story. Houghton Mifflin Harcourt. Boston, Estados Unidos, $324 \mathrm{pp}$.

GDF (2009). Decreto por el que se adicionan diversas disposiciones de la Ley de Residuos Sólidos del Distrito Federal. Gobierno del Distrito Federal. Publicado en la Gaceta Oficial del Distrito Federal el 19 de agosto de 2009.

GDF (2010). Decreto por el que se reforman los artículos $3,6,7,8,10,11,11$ Bis, 14, 18, 23, 25, 26 Bis, 26 Bis 1, 33, 33 Bis, 33 Bis 1, 38, 45, 46, 55 y 69 de la Ley de Residuos Sólidos del Distrito Federal. Gobierno del Distrito Federal. Publicado en la Gaceta Oficial del Distrito Federal el 26 de noviembre de 2010.

Grupo de residuos sólidos - Pontificia Universidad Católica de Valparaíso (2006). Estudio de caracterización de residuos sólidos domiciliarios en la región metropolitana. Reporte. Valaparaíso, Chile, $118 \mathrm{pp}$.

Gutberlet J. y Baeder A. (2008). Informal recycling and occupational health in Santo André, Brazil. Int. J. Environ. Heal. R. 18 (1), 1-15. http://dx.doi. org/10.1080/09603120701844258

Gutiérrez V. (2009). Diagnóstico de residuos sólidos en Bolivia. Ministerio de Medio Ambiente y Agua. Cochabamba, Bolivia, $21 \mathrm{pp}$.

Hopewell J., Dvorak R. y Kosior, E. (2009). Plastics recycling challenges and opportunities. Philos. T. Roy. Soc. A. 364 (1526), 2115-26.

DOI: $10.1098 /$ rstb.2008.0311

INEGI (2012). Anuario estadístico de los Estados Unidos Mexicanos (2011). Instituto Nacional de Estadística y Geografía. México, 155 pp.

Instituto Costarricense de Turismo (2013). El manejo de los residuos en Costa Rica. San José, 64 pp.

IPEA (2012). Plano nacional de Residuos Sólidos: diagnóstico dos residuos urbanos. [en línea]. http://www. mma.gov.br/images/arquivo/80063/Residuos SolidosIPEA.pdf 3/03/2014

IRAM (2009). IRAM 29240 Materiales plásticos biodegradables y/o compostables. Instituto Argentino de Normalización y Certificación. Terminología.

IRAM (2011). IRAM 29421 - Calidad ambiental. Materiales y productos biodegradables y compostables. Requisitos para su valoración mediante compostaje. Instituto Argentino de Normalización y Certificación. $17 \mathrm{pp}$.

Instituto de Ingeniería Sanitaria - Facultad de ingeniería - Universidad de Buenos Aires (2011). Estudio de calidad de los residuos sólidos urbanos del área metropolitana de Buenos Aires - Tercer informe de avance. Reporte. Buenos aires, $106 \mathrm{pp}$.

Instituto Nacional de Estadística (2006). Situación de la generación y manejo de residuos sólidos en Venezuela. Reporte. Caracas, 8 pp. 
Jefatura del estado (2011). Ley 22/2011 de residuos y suelos contaminados. Publicada en el Boletín Oficial Del Estado el 29 de julio de 2011. Madrid, 52 pp.

Karak T., Bhagat R. M. y Bhattacharyya P. (2012). Municipal solid waste generation, composition, and management: the world scenario. Crit. Rev. Env. Sci. Tec., 42 (15), 1509-1630. http://dx.doi.org/10.1080/1 0643389.2011 .569871

Khoo H. H. y Tan R. B. H. (2010). Environmental impacts of conventional plastic and bio-based carrier bags. The International Journal of Life Cycle Assessment 15 (4), 338-345. DOI:10.1007/s11367-010-0162-9

Lazar B. y Gračan R. (2011). Ingestion of marine debris by loggerhead sea turtles, Caretta caretta, in the Adriatic Sea. Mar. Pollut. Bull. 62(1), 43-47. http://dx.doi. org/10.1016/j.marpolbul.2010.09.013

Legislatura de la Ciudad Autónoma de Buenos Aires. (2005). Ley No. 1.854 - Gestión integral de residuos sólidos urbanos. Publicada el 4 de enero de 2006.

Masverde (2011). Venezuela: nueva ordenanza regulara el uso de bolsas plásticas en Falcón. [en línea]. http:// noticias.masverdedigital.com/2011/venezuela-nuevaordenanza-regulara-el-uso-de-bolsas-plasticas-enfalcon/ 10/09/2014

Medina M. (2000). Scavenger cooperatives in Asia and Latin America. Resour. Conserv. Recy. 31 (1) 51-69. http://dx.doi.org/10.1016/S0921-3449(00)00071-9

Medina M. (2005). Serving the unserved: informal refuse collection in Mexico. Waste Manage. Res. 23 (5) 390397. DOI: $10.1177 / 0734242 X 05057698$

Ministerio de agricultura alimentación y medio ambiente (2012). Banco público de indicadores ambientales. Generación de residuos urbanos. [en línea]. Disponible en http://www.magrama.gob.es/es/calidad-y-evaluacion-ambiental/temas/informacion-ambiental-indicadores-ambientales/BPIA_2012_Ficha_web_ResiduosGeneracion_tcm7-164684.pdf $\overline{4} / 08 / 2015$

Ministerio de Agricultura Alimentación y Medio Ambiente (2014). Diagnóstico del sector residuos en España. Análisis Y Perspectiva - Serie Medio Ambiente. Reporte. Madrid, $6 \mathrm{pp}$.

Ministerio del ambiente (2014). Cifras ambientales 2014. Lima, Perú, 13 pp.

Ministerio de Fomento, Ministerio de Agricultura Alimentación y Medio Ambiente, y Centro de Estudios y experimentación de obras públicas en España (2013). Residuos plásticos. Ficha técnica. Madrid, 2013.

Ministerio del Medio Ambiente (2011). Informe del Estado del Medio Ambiente. Reporte. Santiago de Chile, 512 pp.

Ministerio del Medio Ambiente (2014). Sistema nacional de declaración de residuos (SINADER). [en línea]. http:/vu.mma.gob.cl/Documentos/Capacita-
cion_RETC_VU_SINADER_version_publicada.pdf $2 / 02 / 2015$

Müller C., Townsend, K. y Matschullat J. (2012). Experimental degradation of polymer shopping bags (standard and degradable plastic, and biodegradable) in the gastrointestinal fluids of sea turtles. Sci. Total Environ. 416, 464-467. http://dx.doi.org/10.1016/j. scitotenv.2011.10.069

Municipalidad de Pucón (2013). Decreto Exento No. 3140 - Sustitución de bolsas plásticas. Publicado el 7 de enero de 2014. Pucón, Chile, 3pp.

Münnich K., Mahler C. F. y Fricke K. (2006). Pilot project of mechanical-biological treatment of waste in Brazil. Waste Manage. 26 (2), 150-157. http://dx.doi. org/10.1016/j.wasman.2005.07.022

Niezen C. (2014). Bolsas plásticas: más de 3000 mlls se consumen al año en Lima. [en línea]. http://elcomercio. pe/economia/peru/bolsas-plasticas-mas-3-mlls-seconsumen-cada-ano-lima-noticia-1726545 3/05/2014

Observatorio nacional para la gestión de residuos sólidos urbanos (2009). Estadísticas. Generación per cápita y total por provincia y país. [en línea]. http://www. ambiente.gob.ar/observatoriorsu/informacion_general/ estadisticas.html 4/08/2015

OIT y AVINA (2013). Políticas públicas para la inclusión de los recicladores de base al sistema de gestión de residuos municipales en Chile. Organización internacional del trabajo. Reporte. Santiago de Chile, 53 pp.

OPS - Organización Panamericana de la Salud y OMS - Organización Mundial de la Salud (2001). Análisis sectorial de residuos sólidos en Paraguay. Reporte. $267 \mathrm{pp}$.

Osinga S. (2011). Emergent results of artificial economics. Springer Science and Business Media. Nueva York $212 \mathrm{pp}$.

PETSTAR (n.d.). Video institucional. Fecha de consulta: [en línea]. http://www.petstar.com.mx/ 28/04/2015

Plastics Europe (2013). Plastics - the Facts 2013. Reporte. Bruselas, 40 pp.

Plastivida. Monitamento dos índices de reciclagem mecanica de plástico no Brasil (IRmP). Instituto Sócio-Ambiental do Plásticos. [en línea]. http://www.plastivida. org.br/2009/pdfs/IRmP/Apresentacao_IRMP2011.pdf $15 / 04 / 2015$

Poder Ejecutivo - Ciudad de Buenos Aires. (2012). Decreto 180/2012.

Presidencia de la república (1992). Decreto No. 2.216 Normas para el manejo de los desechos sólidos de origen doméstico, comercial, industrial o de cualquier otra naturaleza que no sean peligrosos. Publicado en la Gaceta Oficial el 27 de abril de 1992. Caracas, 1992.

Presidencia de la república (2006). Decreto No. 5.940 Separación de residuos. Brasilia, Brasil, 2 pp. 
Presidencia de la república (2010). Ley No. 12.305 Política Nacional de residuos sólidos. Brasilia, Brasil.

Presidencia de la república de Perú (2010a). Ley que regula las actividades de los recicladores. Lima, pp.

Presidencia de la república de Perú (2010b). Decreto supremo no. 011-2010-MINAM. Medidas de ecoeficiencia para el sector público. Publicado el 26 de agosto de 2010. Lima, Perú.

Presidencia de la República de Uruguay (2010). Decreto 315/10 Compras del Estado a proveedores inscritos en DINAMA y con plan de gestión de recursos. Publicado en el Diario Oficial el 27 de octubre de 2010. Montevideo, 2pp.

Ríos L. M., Moore C. y Jones P. R. (2007). Persistent organic pollutants carried by synthetic polymers in the ocean environment. Mar. Pollut. Bull. 54 (8), 1230-7. http://dx.doi.org/10.1016/j.marpolbul.2007.03.022

Romero O. A. (2014). Proyecto de Ley: Sistema de gestión de envases y residuos de envases. Expediente 4435-D2014. [en línea]. http://www1.hcdn.gov.ar/proyxml/expediente.asp? fundamentos $=$ siynumexp $=4435-\mathrm{D}-2014$ $10 / 09 / 2014$

Schwarz O. (2002). Ciencia de los plásticos. Editorial Costa Nogal. Montevideo, Uruguay, 299 pp.

Scott G. (2011). Venezuela: pueblo prohíbe las bolsas plásticas. [en línea]. http://www.veoverde. com/2011/08/venezuela-pueblo-prohibe-las-bolsasplasticas/ 10/09/2014

Secretaría de Ambiente y Desarrollo Sustentable de la Nación (2012). Sistema de indicadores de desarrollo sostenible - Sexta edición. Buenos Aires, 164 pp.

SEDEMA (2011). Acuerdo por el que se expiden los criterios y normas de producción y consumo sustentable de los productos plásticos. Secretaría del Medio Ambiente. Publicado en la Gaceta Oficial del Distrito Federal el 26 de julio de 2011. Distrito Federal, México, 13 pp.

SEGOB (2014). NMX-E-260-CNCP-2013 Industria del plástico - Materiales bioplásticos - Terminología. Secretaría de Gobernación. Publicada en el Diario Oficial de la Federación el 7 de mayo de 2014.

SEMARNAT (2003). Ley general para la prevención y gestión integral de los residuos. Secretaría de Medio Ambiente y Recursos Naturales. Publicada en el Diario oficial el 8 de octubre de 2003. Distrito Federal, 27 pp.

SEMARNAT (2011). NOM-161-SEMARNAT-2011 Que establece los criterios para clasificar a los Residuos de Manejo Especial y determinar cuáles están sujetos a Plan de Manejo; el listado de los mismos, el procedimiento para la inclusión o exclusión a dicho listado; así como los elementos y procedimientos para la formulación de los planes de manejo. Publicada en el Diario Oficial el 1 de febrero de 2013.
SEMARNAT (2013). Diagnóstico Básico para la gestión integral de los residuos 2012. Secretaría de Medio Ambiente y Recursos Naturales. Distrito Federal, México, 53 pp.

Senado y Cámara de Representantes de la República Oriental del Uruguay (2004). Ley No 17.849 Uso de Envases no retornables. Publicada en el Diario Oficial el 28 de diciembre de 2004. Montevideo.

Terraza H. y Sturzenegger G. (2010). Dinámicas de organización de recicladores informales, tres casos de estudio en América Latina. Reporte del Banco Interamericano de Desarrollo. 65pp.

Themelis N. J. y Diaz Barriga M. E. (2012). Estudio de prefactibilidad técnica y económica para la instalación de capacidad de generación de energía a partir de residuos (WTE) en Uruguay. Reporte. Montevideo, Uruguay, $122 \mathrm{pp}$.

Themelis N. J., Díaz Barriga M. E., Estévez P., y Gaviota Velasco, M. (2013). Guidebook for the application of waste to energy technologies in Latin America and the Caribbean. Earth Engineering Center, Columbia University. 228 pp.

Tolinski M. (2011). Plastics and Sustainability: Towards a Peaceful Coexistence between Bio-based and Fossil Fuel-based Plastics. Wiley-Scrivener. Salem, Massachusetts, $296 \mathrm{pp}$.

Troschinetz A. M. y Mihelcic J. R. (2009). Sustainable recycling of municipal solid waste in developing countries. Waste Manage. 29 (2), 915-23. http://dx.doi. org/10.1016/j.wasman.2008.04.016

UE (2003). Directiva 2002/96/CE sobre los residuos. Parlamento Europeo y Consejo de la Unión Europea. Publicada en el Diario Oficial de la Unión Europea el 27 de enero de 2003.

UE (2008). Directiva 2008/98/CE sobre los residuos. Parlamento Europeo y Consejo de la Unión Europea. Publicada en el Diario Oficial de la Unión Europea el 22 de noviembre de 2008. 28 pp.

United Nations Environment Programme (n.d.). Solid waste management in Rio de Janeiro. [en línea]. http:// waste.ccac-knowledge.net/sites/default/files/CCAC images/city_fact_sheet/Rio_MSW_FactSheet_0.pdf $7 / 08 / 2015$

University of Leeds, International Solid Waste Association, Sweepnet, Wiert y SWAPI (2014). Waste atlas. [en línea]. http://www.atlas.d-waste.com/ 10/09/2014

Vallester E. N. (2010). Gestión de residuos sólidos: impacto sobre los ecosistemas hídricos y áreas costeras. [en línea]. http://www.pnuma.org/aguamiaac/REGIONAL/MATERIAL\%20ADICIONAL/ PRESENTACIONES/PONENTES/Tema $\% 204 \% 20$ -\%20Armonizacion/Gestion\%20de\%20residuos $\% 20$ 
solidos\%20-\%20Erick\%20Vallester/DESECHOS\%20 SOLIDOS\%20PANAMA.pdf 3/03/2014

Van Franeker J. y Law K. (2015). Seabirds, gyres and global trends in plastic pollution. Environ. Pollut. 203, 89-96. DOI: 10.1016/j.envpol.2015.02.034

Vaughn J. (2008). Waste management - A reference handbook. ABC-Clio. California, $311 \mathrm{pp}$.
Viceministerio de gestión ambiental (2012). Cuarto informe nacional de residuos sólidos municipales y no municipales. Reporte. Lima, 379 pp.

Williams R., Ashe E. y O'Hara P. D. (2011). Marine mammals and debris in coastal waters of British Columbia, Canada. Mar. Pollut. Bull. 62 (6), 1303-16. http:// dx.doi.org/10.1016/j.marpolbul.2011.02.029 